\title{
67. PHASE CHEMISTRY STUDIES ON GABBRO AND PERIDOTITE ROCKS FROM SITE 334, DSDP LEG 37
}

\author{
R.F. Symes, J.C. Bevan, and R. Hutchison, \\ Department of Mineralogy, British Museum (Natural History), London, England
}

\section{INTRODUCTION}

Site 334 was drilled on a steep east-facing slope in a small deep basin at $37^{\circ} 2.13^{\prime} \mathrm{N}, 34^{\circ} 24.87^{\prime} \mathrm{W}$. This site lies west of the median valley of the French-American Mid-Ocean Undersea Study area of the Mid-Atlantic Ridge and southwest of the Azores.

Acoustic basement lies beneath 259.5 meters of Recent to late Miocene ooze. The drilled basement consists of an upper 50-meter section of largely aphyric basalts and a lower 67 meters consisting of a complex interlayered sequence of gabbroic and serpentinized peridotite rocks. The rock types found in this unexpectedly high level plutonic series comprise coarse gabbros, - serpentinized olivine gabbros, serpentinized plagioclase peridotites, and sedimentary and tectonic breccias. A brief summary of the main petrological features of the 10 rock specimens investigated (in order of depth) is given in Table 1.

The unaltered silicate phases and spinel group minerals in 10 specimens from the plutonic complex have been analyzed by electron microprobe, and the results are shown in Tables 2-7. Where feldspar and pyroxene phases occur, core and rim analyses have been made to detect zoning. The compositions of the fine exsolution lamellae developed in many of the analyzed pyroxenes could not be determined. Phases showing strong alteration and sulfide minerals have not been analyzed.

\section{TEXTURES AND PHASE CHEMISTRY}

\section{Plagioclase}

Most sections have twinned plagioclase in differing stages of alteration to a clay mineral aggregate. Frequently, small euhedral to subhedral feldspars are enclosed poikilitically in pyroxene or olivine, but have compositions very similar to the larger and more irregular grains which predominate in these rocks. Where zonation, not always optically apparent, occurs, it is reversed with narrow rims having increased contents of An. Apart from the eucritic rock 22-1, 17-23 $\mathrm{cm}$, the rocks studied contain plagioclase with compositions more calcic than Anso (Figure 3). Compositions do not vary directly with depth of the rock.

\section{Olivine}

Olivine is strongly serpentinized in most thin sections studied, in a similar way to the serpentinized ultramafic intrusions from the Mid-Atlantic Ridge described by Aumento and Loubat (1971). However, ovoids of unaltered olivine occur within the serpentine. These ovoids are often in optical continuity indicating an original large grain size. The olivines analyzed have a narrow range of composition: Fo85-89 (Figure 2).

\section{Pyroxene}

Both clinopyroxenes and orthopyroxenes have been analyzed in the rocks. Grains enclosed in plagioclase have compositions similar to those which in turn enclose feldspar. Alteration to green or brown (clinopyroxene) or brown (orthopyroxene) material, similar in composition (for oxides other than $\mathrm{SiO}_{2}$ ) to that of the original pyroxene, is widespread, the orthopyroxene being the first to break down. Narrow exsolution lamellae are also common, but could not be resolved: the compositions given of grains showing exsolution represent bulk analyses of both lamellae and matrix.

Some grains have narrow diopsidic rims surrounding cores which, with the exception of the augite of Sample $22-1,17-23 \mathrm{~cm}$, all plot in the endiopside field (Figure 1). The augite appears as relict grains in optical continuity within a replacing green amphibole, which follows the original pyroxene orientation.

\section{Chromite}

The chromite spinels are euhedral or subhedral to rounded, and occasionally have apophyses along grain boundaries. They occur within and between both

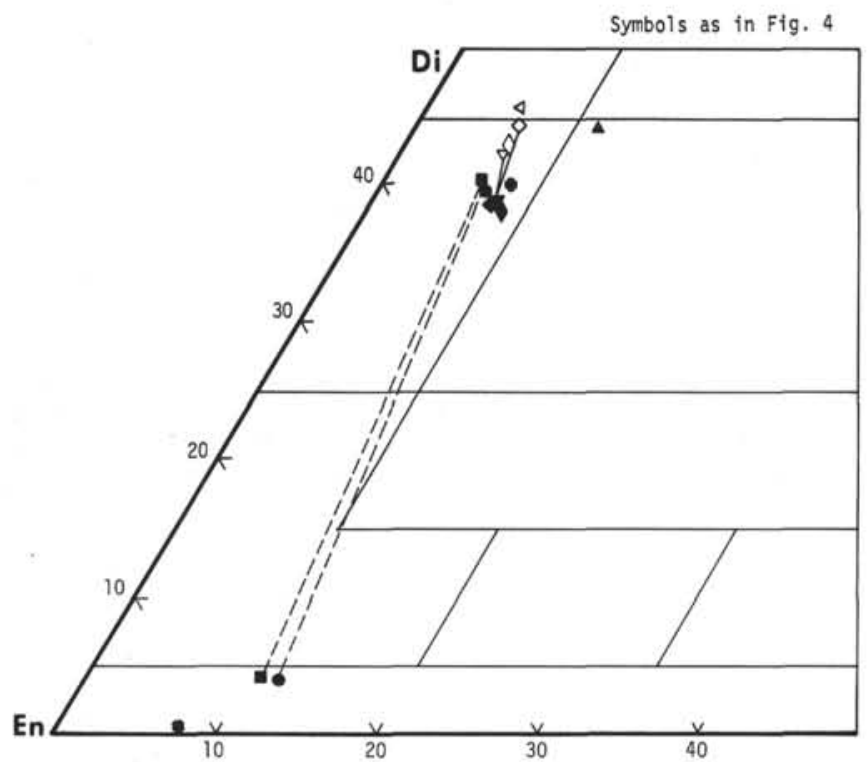

Figure 1. Pyroxene compositions for gabbros and peridotites from Site 334. 
TABLE 1

Summary of the Petrology at Site 334

\begin{tabular}{|c|c|c|c|c|c|c|c|c|}
\hline $\begin{array}{l}\text { Sample } \\
\text { (Interval } \\
\text { in } \mathrm{cm} \text { ) }\end{array}$ & Rock Type & Texture & Plagioclase & Olivine & Clinopyroxene & Orthopyroxene & Chromite & Ore Minerals \\
\hline $\begin{array}{l}22-1 \\
17-23\end{array}$ & Eucrite & $\begin{array}{l}\text { Coarse- } \\
\text { grained } \\
\text { cumulate } \\
\text { showing } \\
\text { cataclasia }\end{array}$ & $\begin{array}{l}\text { Cumulus; granulated } \\
\text { at margins }\left(\mathrm{An}_{79}\right)\end{array}$ & & $\begin{array}{l}\text { Colorless; cumulus } \\
\text { now relict in green } \\
\text { pleochroic } \\
\text { amphibole } \\
\left(\mathrm{Fs}_{12} \mathrm{En}_{44} \mathrm{Wo}_{44}\right)\end{array}$ & $\begin{array}{l}\text { Intercumulus; } \\
\text { completely altered }\end{array}$ & & $\begin{array}{l}\text { Magnetite: orientated } \\
\text { flecks in amphibole; } \\
\text { dust trails in feldspar }\end{array}$ \\
\hline $\begin{array}{l}22-2 \\
80-82\end{array}$ & Peridotite & $\begin{array}{l}\text { Coarse- } \\
\text { grained } \\
\text { cumulate }\end{array}$ & $\begin{array}{l}\text { Partially altered to } \\
\text { a clay mineral } \\
\left(\mathrm{An}_{88}\right)\end{array}$ & $\begin{array}{l}\text { Cumulus; partially } \\
\text { serpentinized } \\
\left(\mathrm{Fo}_{89}\right)\end{array}$ & $\begin{array}{l}\text { Colorless; cumulus; } \\
\text { exsolution lamellae } \\
\left(\mathrm{Fs}_{6} \mathrm{En}_{53} \mathrm{Wo}_{40}\right)\end{array}$ & $\begin{array}{l}\text { Colorless; cumulus } \\
\left(\mathrm{Fs}_{11} \mathrm{En}_{85} \mathrm{Wo}_{4}\right)\end{array}$ & $\begin{array}{l}\text { Large, eu- to sub- } \\
\text { hedral; reddish- } \\
\text { brown; altered; } \\
\text { contains inclusions }\end{array}$ & $\begin{array}{l}\text { Iron oxides in } \\
\text { serpentine; as halos } \\
\text { to chromite }\end{array}$ \\
\hline $\begin{array}{l}23-1 \\
46-54\end{array}$ & Peridotite & $\begin{array}{l}\text { Very } \\
\text { coarse } \\
\text { grained } \\
\text { cumulate }\end{array}$ & $\begin{array}{l}\text { Altered to a clay } \\
\text { mineral }\left(\mathrm{An}_{>90}\right)\end{array}$ & $\begin{array}{l}\text { Large, partially } \\
\text { serpentinized; } \\
\text { cumulus }\left(\mathrm{Fo}_{89} \text { ) }\right.\end{array}$ & $\begin{array}{l}\text { Colorless; cumulus; } \\
\text { exsolution lamellae } \\
\left(\mathrm{Fs}_{6} \mathrm{En}_{48} \mathrm{Wo}_{46}\right)\end{array}$ & $\begin{array}{l}\text { Intercumulus; } \\
\text { completely altered }\end{array}$ & $\begin{array}{l}\text { Rounded; reddish- } \\
\text { brown; altered }\end{array}$ & $\begin{array}{l}\text { Iron oxides in } \\
\text { serpentine; rims and } \\
\text { halos to chromite }\end{array}$ \\
\hline $\begin{array}{l}23-1 \\
91-93\end{array}$ & Allivalite & $\begin{array}{l}\text { Medium- } \\
\text { grained } \\
\text { brecciated } \\
\text { cumulate }\end{array}$ & $\begin{array}{l}\text { Cumulus; granulated } \\
\left(\mathrm{An}_{85}\right)\end{array}$ & & $\begin{array}{l}\text { Colorless; cumulus; } \\
\text { exsolution lamellae; } \\
\text { partially altered } \\
\left(\mathrm{Fs}_{8} \mathrm{En}_{52} \mathrm{Wo}_{40}\right)\end{array}$ & Completely altered & & Rare magnetite clots \\
\hline $\begin{array}{l}23-2 \\
78-82\end{array}$ & Peridotite & $\begin{array}{l}\text { Coarse- } \\
\text { grained } \\
\text { cumulate }\end{array}$ & $\begin{array}{l}\text { Rare; altered in } \\
\text { altered pyroxene }\end{array}$ & All serpentinized & Completely altered & Completely altered & $\begin{array}{l}\text { Euhedral to } \\
\text { rounded; altered }\end{array}$ & $\begin{array}{l}\text { Iron oxides from } \\
\text { altered spinel }\end{array}$ \\
\hline $\begin{array}{l}24-2 \\
105-107\end{array}$ & Allivalite & $\begin{array}{l}\text { Coarse- } \\
\text { grained } \\
\text { cumulate }\end{array}$ & $\begin{array}{l}\text { Cumulus; some } \\
\text { euhedral }\left(\mathrm{An}_{91}\right)\end{array}$ & $\begin{array}{l}\text { Rare, cumulus; all } \\
\text { serpentinized }\end{array}$ & $\begin{array}{l}\text { Cumulus, adcumulus; } \\
\text { exsolution lamellae } \\
\left(\mathrm{Fs}_{6} \mathrm{En}_{54} \mathrm{Wo}_{40}\right)\end{array}$ & $\begin{array}{l}\text { Colorless; altered } \\
\text { margins } \\
\left(\mathrm{Fs}_{12} \mathrm{En}_{84} \mathrm{Wo}_{4}\right)\end{array}$ & $\begin{array}{l}\text { Rare; orange- } \\
\text { brown }\end{array}$ & $\begin{array}{l}\text { Magnetite dust trails in } \\
\text { all phases; composite } \\
\text { grains with sulfide }\end{array}$ \\
\hline $\begin{array}{l}24-4 \\
100-106\end{array}$ & Allivalite & $\begin{array}{l}\text { Coarse- } \\
\text { grained } \\
\text { cumulate }\end{array}$ & $\begin{array}{l}\text { Cumulus, adcumulus; } \\
\text { some euhedral } \\
\left(\mathrm{An}_{89} ; \operatorname{rim} \mathrm{An}_{91}\right)\end{array}$ & $\begin{array}{l}\text { Cumulus; partially } \\
\text { serpentinized } \\
\left(\mathrm{Fo}_{86}\right)\end{array}$ & $\begin{array}{l}\text { Colorless; cumulus; } \\
\text { exsolution lamellae; } \\
\text { optical zoning } \\
\left(\mathrm{Fs}_{8} \mathrm{En}_{53} \mathrm{Wo}_{39} ; \mathrm{rim}\right. \\
\left.\mathrm{Fs}_{5} \mathrm{En}_{51} \mathrm{Wo}_{42}\right)\end{array}$ & & $\begin{array}{l}\text { Rounded; in } \\
\text { clinopyroxene }\end{array}$ & $\begin{array}{l}\text { Iron oxides in } \\
\text { serpentine; rare } \\
\text { composite clots }\end{array}$ \\
\hline $\begin{array}{l}26-1 \\
70-75\end{array}$ & Harzburgite & $\begin{array}{l}\text { Coarse- } \\
\text { grained } \\
\text { cumulate }\end{array}$ & $\begin{array}{l}\text { Rare; completely } \\
\text { altered }\end{array}$ & All serpentinized & $\begin{array}{l}\text { Inclusion in } \\
\text { chromite }\end{array}$ & $\begin{array}{l}\text { Altered grains with } \\
\text { exsolution lamellae; } \\
\left(\mathrm{Fs}_{7} \mathrm{En}_{93} \mathrm{Wo}_{0}\right) \\
\text { inclusions in } \\
\text { chromite }\end{array}$ & $\begin{array}{l}\text { Large, eu- to sub- } \\
\text { hedral; reddish- } \\
\text { brown; black } \\
\text { alteration; } \\
\text { inclusions }\end{array}$ & $\begin{array}{l}\text { Magnetite: as diffuse } \\
\text { halos to olivine, } \\
\text { pyroxene, chromite; } \\
\text { trails in orthopyroxene }\end{array}$ \\
\hline $\begin{array}{l}26-2 \\
56-71\end{array}$ & Allivalite & $\begin{array}{l}\text { Coarse- } \\
\text { grained } \\
\text { cumulate }\end{array}$ & $\begin{array}{l}\text { Cumulus, inter- } \\
\text { cumulus; some } \\
\text { euhedral } \\
\left(\mathrm{An}_{87} ; \mathrm{rim} \mathrm{An}_{91}\right)\end{array}$ & $\begin{array}{l}\text { Cumulus; partially } \\
\text { serpentinized } \\
\left(\mathrm{Fo}_{85}\right)\end{array}$ & $\begin{array}{l}\text { Colorless; cumulus; } \\
\text { exsolution lamellae } \\
\left(\mathrm{Fss}_{8} \mathrm{En}_{54} \mathrm{Wo}_{39} ;\right. \\
\left.\text { rim } \mathrm{Fs}_{7} \mathrm{En}_{49} \mathrm{Wo}_{44}\right)\end{array}$ & & $\begin{array}{l}\text { Rare; small; } \\
\text { rounded; in } \\
\text { pyroxene }\end{array}$ & $\begin{array}{l}\text { Rare; composite } \\
\text { sulfide/spinel group } \\
\text { clots }\end{array}$ \\
\hline $\begin{array}{l}26-2 \\
59-64\end{array}$ & Allivalite & $\begin{array}{l}\text { Coarse- } \\
\text { grained } \\
\text { cumulate }\end{array}$ & $\begin{array}{l}\text { Cumulus } \\
\left(\mathrm{An}_{91} ; \operatorname{rim} \mathrm{An}_{93}\right)\end{array}$ & $\begin{array}{l}\text { Cumulus; partially } \\
\text { serpentinized } \\
\left(\text { Fog6 }_{86}\right)\end{array}$ & $\begin{array}{l}\text { Colorless; cumulus; } \\
\text { exsolution lamellae; } \\
\text { twinning } \\
\mathrm{Fs}_{8} \mathrm{En}_{54} \mathrm{Wo}_{39} ; \\
\operatorname{rim~} \mathrm{Fs}_{7} \mathrm{En}_{50} \mathrm{Wo}_{43} \text { ) }\end{array}$ & & $\begin{array}{l}\text { Small; rounded; } \\
\text { in pyroxene }\end{array}$ & $\begin{array}{l}\text { Iron oxides in } \\
\text { serpentine; rare sulfides }\end{array}$ \\
\hline
\end{tabular}


TABLE 2

Analyses of Plagioclase Feldspars

\begin{tabular}{|c|c|c|c|c|c|c|c|c|}
\hline & \multirow[b]{2}{*}{$\begin{array}{l}22-1 \\
17-23\end{array}$} & \multirow[b]{2}{*}{$\begin{array}{l}22-2 \\
80-82\end{array}$} & \multirow[b]{2}{*}{$\begin{array}{c}23-1 \\
46-54 \\
\text { (Altered) }\end{array}$} & \multirow[b]{2}{*}{$\begin{array}{l}23-1 \\
91-93\end{array}$} & \multirow[b]{2}{*}{$\begin{array}{c}24-2, \\
105-107\end{array}$} & \multicolumn{2}{|c|}{ Sample (Interval in $\mathrm{cm}$ ) } & \multirow[b]{2}{*}{$\begin{array}{c}26-2, \\
59-64 \\
\text { Core/Rim }\end{array}$} \\
\hline & & & & & & $\begin{array}{c}24-4, \\
100-106 \\
\text { Core/Rim }\end{array}$ & $\begin{array}{c}26-2, \\
56-71 \\
\text { Core/Rim }\end{array}$ & \\
\hline $\mathrm{SiO}_{2}$ & 47.99 & 45.44 & & 46.59 & 46.54 & $46.22 / 45.53$ & $46.62 / 46.17$ & $45.93 / 45.82$ \\
\hline $\mathrm{Al}_{2} \mathrm{O}_{3}$ & 33.34 & 35.71 & & 31.67 & 33.48 & $32.93 / 33.04$ & $33.54 / 33.43$ & $34.82 / 34.67$ \\
\hline $\mathrm{CaO}^{2}$ & 16.06 & 18.45 & & 17.69 & 18.08 & $18.32 / 18.20$ & $18.11 / 18.40$ & $18.31 / 18.82$ \\
\hline $\mathrm{Na}_{2} \mathrm{O}$ & 2.30 & 1.32 & & 1.73 & 1.09 & $1.26 / 1.03$ & $1.45 / 1.06$ & $1.25 / 1.24$ \\
\hline $\mathrm{K}_{2} \mathrm{O}$ & 0.01 & 0.05 & & 0.02 & 0.01 & $0.02 / 0.02$ & $0.00 / 0.00$ & $0.01 / 0.01$ \\
\hline Total & 99.70 & 100.97 & & 97.70 & 99.20 & $98.75 / 97.82$ & $99.72 / 99.06$ & $100.32 / 100.56$ \\
\hline an & 79.4 & 88.3 & $\begin{array}{l}\text { (approx. } \\
>90 \text { ) }\end{array}$ & 84.9 & 90.2 & $88.8 / 90.6$ & $87.4 / 90.6$ & $89.0 / 89.3$ \\
\hline$a b$ & 20.6 & 11.4 & & 15.0 & 9.8 & $11.1 / 9.3$ & $12.6 / 9.4$ & $11.0 / 10.7$ \\
\hline or & 0.1 & 0.3 & & 0.1 & 0.1 & $0.1 / 0.1$ & $0.0 / 0.0$ & $0.0 / 0.0$ \\
\hline
\end{tabular}

TABLE 3

Olivine Compositions

\begin{tabular}{lccccc}
\hline \multicolumn{5}{c}{ Sample (Interval in cm) } \\
\hline & $22-2$, & $23-1$ & $24-4$, & $26-2$, & $26-2$, \\
& $80-82$ & $46-54$ & $100-106$ & $56-71$ & $59-64$ \\
\hline fa & 11.1 & 11.1 & 14.0 & 14.7 & 14.2 \\
fo & 88.9 & 88.9 & 86.0 & 85.3 & 85.8 \\
\hline
\end{tabular}

pyroxene and serpentinized olivine and may have inclusions which are similar in composition to both clinoand orthopyroxenes elsewhere in the thin section. Irregular bands of a black alteration product, possibly a mixture of oxides and a chlorite, cross the grains, which are commonly rimmed with magnetite and/or a pyroxene. Compositionally, little variation was found among grains in the same section, except in Sample 26$1,70-75 \mathrm{~cm}$, where two groups were distinguished. The grains of Group 1 are larger in size but have smaller $\mathrm{Fe} / \mathrm{Mg}$ ratios than those in Group 2.

Chromite analyses are given in Table 7 and plotted on a spinel-chromite-magnetite diagram in Figure 4. For the purposes of this plot, $\mathrm{Fe}_{2} \mathrm{O}_{3}$ was calculated from total iron assuming an atomic ratio of $\mathrm{R}^{2+}$ to $\mathrm{R}^{3+}$ of $1: 2$. The analyses form a close group in the aluminum chromite field. Also plotted are unpublished microprobe analyses by J.M. Hall of minerals of the spinel group from St Paul Rocks, an upper mantlederived ultrabasic intrusion emplaced largely in the solid state (Melson et al., 1972). This second group lies within the chromian spinel field and shows little affinity with the chromites from Site 334 .

\section{Discussion}

All the rocks studied are plutonic and show varying degrees of serpentinization and brecciation. From the textural evidence, the paragenetic sequence appears to

TABLE 4

Analyses of Clinopyroxenes

\begin{tabular}{|c|c|c|c|c|c|c|c|c|}
\hline & \multicolumn{8}{|c|}{ Sample (Interval in cm) } \\
\hline & $\begin{array}{c}22-1, \\
17-23 \\
\text { Relict Grains } \\
\text { in Amphibole }\end{array}$ & $\begin{array}{l}22-2 \\
80-82\end{array}$ & $\begin{array}{c}23-1 \\
46-54\end{array}$ & $\begin{array}{l}23-1, \\
91-93\end{array}$ & $\begin{array}{c}24-2, \\
105-107\end{array}$ & $\begin{array}{c}24-4, \\
100-106 \\
\text { Core/Rim }\end{array}$ & $\begin{array}{c}26-2, \\
56-71 \\
\text { Core/Rim }\end{array}$ & $\begin{array}{c}26-2 \\
59-64 \\
\text { Core/Rim }\end{array}$ \\
\hline $\mathrm{SiO}_{2}$ & & 52.41 & 50.54 & 50.92 & 52.86 & $52.91 / 52.47$ & $52.87 / 52.96$ & $52.42 / 52.52$ \\
\hline $\mathrm{TiO}_{2}$ & & 0.19 & 0.28 & 0.09 & 0.13 & $0.13 / 0.19$ & $0.15 / 0.19$ & $0.15 / 0.17$ \\
\hline $\mathrm{Al}_{2} \mathrm{O}_{3}$ & & 3.32 & 2.52 & 4.42 & 2.70 & $3.18 / 2.33$ & $3.16 / 2.42$ & $2.80 / 2.74$ \\
\hline $\mathrm{Cr}_{2} \mathrm{O}_{3}$ & & 1.32 & 1.25 & 0.30 & 0.61 & $1.11 / 0.60$ & $0.93 / 0.79$ & $0.70 / 0.53$ \\
\hline $\mathrm{FeO}^{*}$ & & 4.04 & 3.70 & 5.00 & 4.12 & $4.82 / 4.06$ & $4.85 / 4.09$ & $4.97 / 4.30$ \\
\hline $\mathrm{MnO}$ & & 0.17 & 0.14 & 0.14 & 0.06 & $0.16 / 0.17$ & $0.16 / 0.11$ & $0.16 / 0.16$ \\
\hline $\mathrm{MgO}$ & & 19.52 & 17.20 & 18.18 & 19.50 & $18.87 / 18.16$ & $19.18 / 17.36$ & $19.41 / 18.10$ \\
\hline $\mathrm{CaO}$ & & 20.52 & 22.66 & 19.55 & 20.00 & $19.12 / 20.94$ & $19.14 / 21.97$ & $19.49 / 21.55$ \\
\hline $\mathrm{Na}_{2} \mathrm{O}$ & & 0.24 & 0.27 & 0.25 & 0.03 & $0.12 / 0.08$ & $0.07 / 0.11$ & $0.12 / 0.13$ \\
\hline Total & & 101.73 & 98.56 & 98.85 & 100.01 & $100.42 / 99.00$ & $100.51 / 100.00$ & $100.22 / 100.20$ \\
\hline fs & 11.5 & 6.2 & 5.8 & 8.0 & 6.4 & $7.6 / 6.5$ & $7.6 / 6.5$ & $7.7 / 6.7$ \\
\hline en & 44.1 & 53.4 & 48.4 & 51.9 & 53.9 & $53.4 / 51.1$ & $53.8 / 49.0$ & $53.6 / 50.2$ \\
\hline wo & 44.4 & 40.4 & 45.8 & 40.1 & 39.7 & $38.9 / 42.4$ & $38.6 / 44.5$ & $38.7 / 43.0$ \\
\hline
\end{tabular}

Note: $\mathrm{FeO}^{*}=$ total iron. 


$$
\text { Fo- }
$$

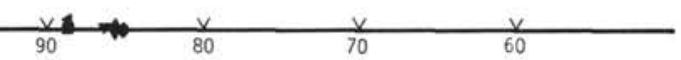

Figure 2. Olivine compositions for gabbros and peridotites from Site 334.

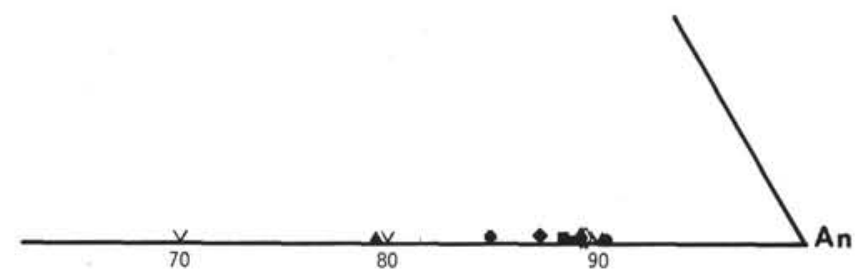

Figure 3. Plagioclase compositions for gabbros and peridotites from Site 334.

TABLE 5

Analyses of Orthopyroxenes

\begin{tabular}{|c|c|c|c|}
\hline & \multicolumn{3}{|c|}{ Sample (Interval in $\mathrm{cm}$ ) } \\
\hline & $\begin{array}{l}22-2 \\
80-82\end{array}$ & $\begin{array}{c}24-2 \\
105-107\end{array}$ & $\begin{array}{c}26-1 \\
70-75 \\
\text { Relict Grains }\end{array}$ \\
\hline $\mathrm{SiO}_{2}$ & 56.15 & 56.15 & \\
\hline $\mathrm{TiO}_{2}$ & 0.11 & 0.07 & \\
\hline $\mathrm{Al}_{2} \mathrm{O}_{3}$ & 2.14 & 1.76 & \\
\hline $\mathrm{Cr}_{2} \mathrm{O}_{3}$ & 0.74 & 0.35 & \\
\hline $\mathrm{FeO}^{*}$ & 7.40 & 8.03 & \\
\hline $\mathrm{MnO}$ & 0.19 & 0.14 & \\
\hline $\mathrm{MgO}$ & 32.87 & 31.90 & \\
\hline $\mathrm{CaO}$ & 2.21 & 2.16 & \\
\hline $\mathrm{Na}_{2} \mathrm{O}$ & 0.00 & 0.00 & \\
\hline Total & 101.81 & 100.56 & \\
\hline fs & 10.7 & 11.9 & 7.3 \\
\hline en & 85.1 & 84.1 & 92.7 \\
\hline wo & 4.1 & 4.1 & 0.1 \\
\hline
\end{tabular}

Note: $\mathrm{FeO}^{*}=$ total iron.

be: precipitation of primary minerals from a melt, followed by accumulation and kink-band formation in olivine, and partial alteration of chrome spinel to magnetite, and later brecciation and granulation of the gabbroic rocks $22-1,17-23 \mathrm{~cm}$ and $23-1,91-93 \mathrm{~cm}$. Alteration products, most of which predate the brecciation, may include the serpentine group, green amphibole, brown and yellow clay minerals, and a low$\mathrm{K}$, micaceous material. Despite serpentinization and later deformation, some textural and compositional similarities exist between the rocks studied and those from the Bushveld and Stillwater complexes. Primary textures are indicative of a cumulate origin and hence the nomenclature of Wager and Brown (1967) can be employed. The uppermost rock, $22-1,17-23 \mathrm{~cm}$, is a plagioclase-pyroxene cumulate.Remaining rocks are allivalites or peridotites.
TABLE 6

Analysis of Amphibole

\begin{tabular}{lc}
\hline & $\begin{array}{c}\text { Sample 22-1, } \\
17-23 \mathrm{~cm} \\
\text { Replacing } \\
\text { Clinopyroxene } \\
\text { (Table 4) }\end{array}$ \\
\hline $\mathrm{SiO}_{2}$ & 44.44 \\
$\mathrm{TiO}_{2}$ & 0.46 \\
$\mathrm{Al}_{2} \mathrm{O}_{3}$ & 7.64 \\
$\mathrm{Cr}_{2} \mathrm{O}_{3}$ & 0.29 \\
$\mathrm{FeO}^{*}$ & 9.65 \\
$\mathrm{MnO}$ & 0.11 \\
$\mathrm{MgO}$ & 15.96 \\
$\mathrm{CaO}$ & 10.83 \\
$\mathrm{Na}{ }_{2} \mathrm{O}$ & 2.00 \\
$\mathrm{~K}_{2} \mathrm{O}$ & 0.22 \\
$\mathrm{Total}$ & 91.60 \\
$(\mathrm{Ti}+\mathrm{Mn}+\mathrm{Fe})$ & 13.4 \\
$(\mathrm{Mg}+\mathrm{Cr})$ & 44.5 \\
$\mathrm{Ca}$ & 19.6 \\
$(\mathrm{Na}+\mathrm{K})$ & 6.5 \\
$\mathrm{Al}$ & 16.0 \\
\hline
\end{tabular}

Note: $\mathrm{FeO}^{*}=$ total iron.

There is an alternation between rocks with and without cumulus plagioclase. The association of orthopyroxene-bearing (i.e., of tholeiitic affinity) mafic and feldspathic cumulates is similar to that on Rhum (Wager and Brown, 1967), where rhythmic units have mafic bases and feldspathic tops. A minimum of four such units may have been sampled here, but alternatively the association may be the result of faulting of one unit. The upper-most peridotite contains the most magnesian olivine and is almost certainly not the basal part of a unit of which the overlying eucrite was the top. The eucritic gabbro appears to form a unit on its own.

\section{ACKNOWLEDGMENTS}

We thank Dr. D.R.C. Kempe for helpful advice during this study and for critically reading the manuscript.

\section{REFERENCES}

Aumento, F. and Loubat, H., 1971. The Mid-Atlantic Ridge near $45^{\circ} \mathrm{N}$ XVI. Serpentinized ultramafic intrusions: Canadian J. Earth Sci., v. 8, p. 631-663.

Melson, W.G., Hart, S.R., and Thompson, G., 1972. St. Paul's Rocks, equatorial Atlantic: Petrogenesis, radiometric ages and implications on sea floor spreading: Geol. Soc. Am. Mem. 132, p. 241-272.

Wager, L.R. and Brown, G.M., 1967. Layered igneous rocks: Edinburgh (Oliver and Boyd.). 


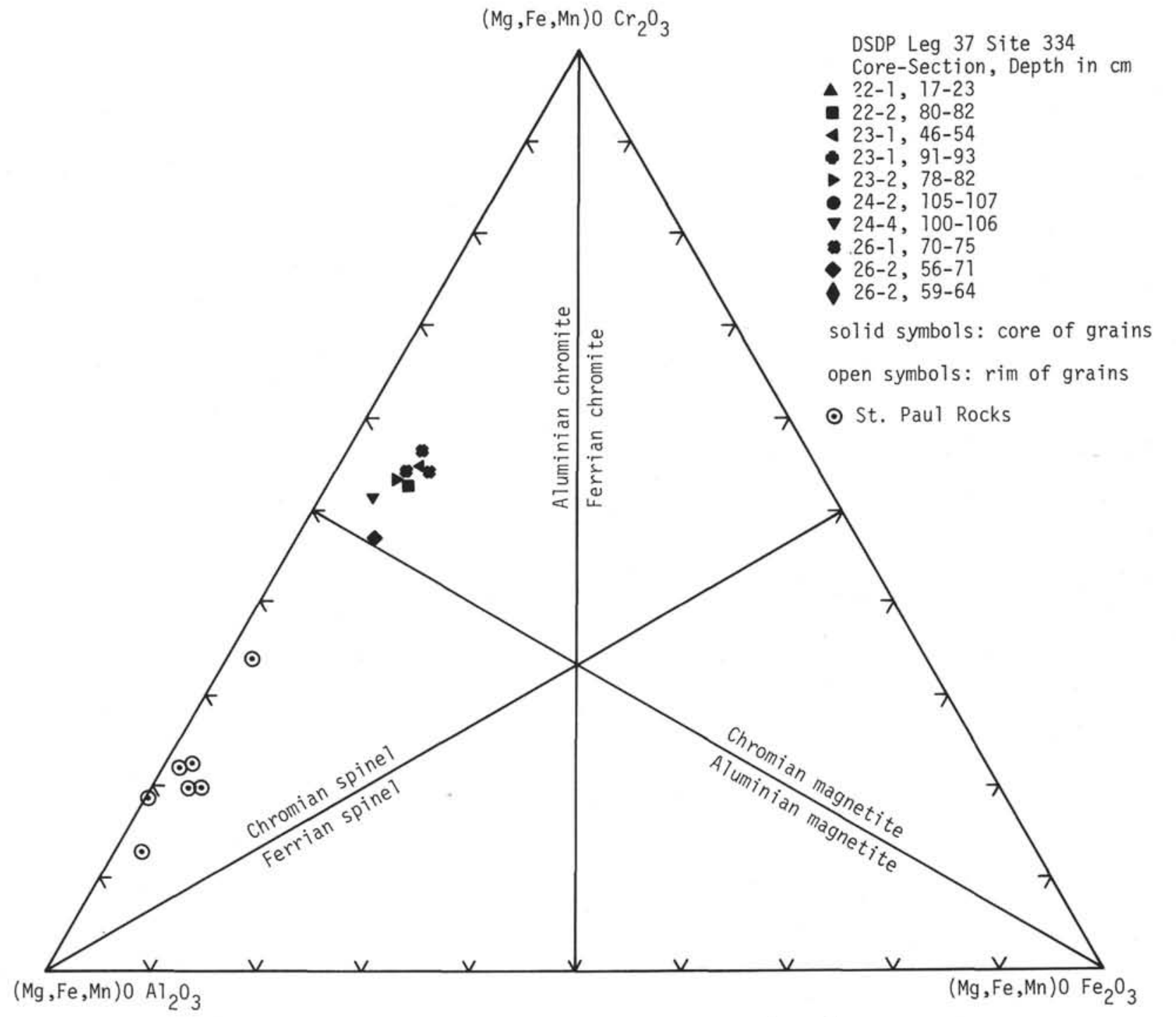

Figure 4. Spinel group compositions $\mathrm{Fe}_{2} \mathrm{O}_{3}$ calculated from total Fe assuming $R^{2+}: R^{3+}=1: 2$ for gabbros and peridotites from Site 334.

TABLE 7

Analyses of Chromites

\begin{tabular}{|c|c|c|c|c|c|c|c|c|}
\hline & & \multicolumn{7}{|c|}{ Sample (Interval in $\mathrm{cm}$ ) } \\
\hline & & & & & & $26-1,70$ & & \\
\hline & $\begin{array}{l}22-2 \\
80-82\end{array}$ & $\begin{array}{r}23-1, \\
46-54\end{array}$ & $\begin{array}{r}23-2 \\
78-82\end{array}$ & $\begin{array}{c}24-4 \\
100-106\end{array}$ & Group 1 & Group 2 & $\begin{array}{l}\text { contains } \\
\text { inclusions }\end{array}$ & $\begin{array}{r}26-2, \\
56-71\end{array}$ \\
\hline $\mathrm{TiO}_{2}$ & 0.65 & 0.59 & 0.45 & 0.46 & 0.61 & 0.49 & 0.53 & 0.20 \\
\hline $\mathrm{Al}_{2} \mathrm{O}_{3}$ & 20.87 & 18.86 & 21.57 & 23.36 & 20.36 & 18.83 & 19.39 & 25.20 \\
\hline $\mathrm{Cr}_{2} \mathrm{O}_{3}$ & 41.92 & 42.21 & 42.53 & 41.32 & 43.44 & 44.01 & 43.53 & 38.98 \\
\hline $\mathrm{FeO}^{*}$ & 24.21 & 22.85 & 19.43 & 22.52 & 20.27 & 23.09 & 20.62 & 23.89 \\
\hline $\mathrm{MnO}$ & 0.31 & 0.32 & 0.30 & 0.29 & 0.27 & 0.21 & 0.28 & 0.27 \\
\hline $\mathrm{MgO}$ & 10.61 & 10.77 & 12.85 & 10.40 & 12.42 & 10.71 & 12.78 & 11.55 \\
\hline Total & 98.57 & 95.60 & 97.13 & 98.35 & 97.37 & 97.34 & 97.13 & 100.09 \\
\hline$(\mathrm{Ti}+\mathrm{Al})$ & 26.5 & 24.8 & 27.1 & 29.2 & 25.9 & 24.3 & 24.7 & 30.4 \\
\hline $\mathrm{Cr}$ & 35.1 & 36.5 & 35.4 & 34.4 & 36.3 & 37.5 & 36.5 & 31.4 \\
\hline$(\mathrm{Fe}+\mathrm{Mn})$ & 21.7 & 21.2 & 17.4 & 20.1 & 18.2 & 21.0 & 18.6 & 20.6 \\
\hline $\mathrm{Mg}$ & 16.7 & 17.5 & 20.1 & 16.3 & 19.6 & 17.2 & 20.2 & 17.6 \\
\hline
\end{tabular}

Note: $\mathrm{FeO}^{*}=$ total iron. 